\title{
Caractéristiques des protéines du fromage précipitées par des ions calcium au cours de l'électrophorèse sur gel d'amidon
}

\author{
par \\ J. RYMASZEWSKI, S. POZNANSKI \\ Université d'Agriculture à Olsztyn (Pologne) \\ Chaire de la Technologie Laitière \\ et B. HABAJ \\ Institut de l'Industrie laitière \\ Laboratoire des Cultures pures lactiques \\ Olsztyn - Kortowo (Pologne)
}

\section{INTRODUCTION}

Il ressort des observations de Ney et coll. (1966) que la méthode de l'électrophorèse sur gel de polyacrylamide est utile pour étudier le cours de l'hydrolyse des protéines du fromage. Ils ont constaté des différences évidentes dans la répartition des fractions peptidiques entre des fromages à moisissure ("bleus"), des fromages à pâte dure et ceux à pâte molle. Assenat (1967) a fait un assez bon usage de ce procédé pour découvrir la présence des protéines du lait de vache dans le Roquefort. Payens (1958) et Poznañski (1964) ont remarqué, en se servant de l'électrophorèse sur papier, que la dégradation de la caséine $\alpha_{\mathrm{s}}$ au cours de l'affinage des fromages (Edam, Tilsit et Trappiste) était plus intense que ne l'était celle de la caséine $\beta$. La même observation a été faite par Christ (1965) qui utilisait l'électrophorèse sur gel de polyacrylamide. Les essais d'Emmons et coll. (1962), ainsi que les nôtres - Poznañski, Lenoir, Mocquot (1965); Poznañski, Rymaszewski (1965) ; Rymaszewski, Poznañski (1968) suggèrent que les enzymes des bactéries lactiques qui entrent dans la composition des levains ne sont pas capables de dégrader la caséine (excepté la para-\% caséine) d'une manière évidente, et qu'ils n'en font qu'hydrolyser des produits intermédiaires de dégradation que l'action de la présure a accumulés. 
Au cours de nos expérimentations actuelles nous allons présenter, à la base de la méthode de l'électrophorèse sur gel d'amidon, la caractéristique du degré de dégradation des protéines du fromage insolubles dans la solution de $0,03 \mathrm{M}$ des ions de calcium. Notre analyse concerne des fromages affinés, fabriqués au moyen de quelques monocultures ou de leurs cultures mixtes.

\section{EXPERIMENTATION}

\section{Renseignement sur les fromages analysés}

La fraction des protéines de fromages de la sorte d'Edam, insoluble dans la solution de $0,03 \mathrm{M}$ du chlorure de calcium, a été analysée au cours de nos investigations. Des lots différents de lait de fromagerie étaient ensemencés avec du levain de cultures pures de bactéries lactiques ou de leurs cultures mixtes: Str. lactis 134 et 136 , Str. diacetilactis 216 et 222 , la culture-mixte de ces quatre souches $\left(\mathrm{M}_{1}\right)$, Strept. Thermophilus et $L$. helveticus et leur culture mixte $\left(\mathrm{M}_{2}\right)$. Les essais étaient répétés deux fois. Dans la première expérimentation, une quantité assez grande de lactose a été laissée exprès dans la pâte du fromage pour assurer le maintien du $p H$ bas dans le fromage au cours de trois mois d'affinage. A partir du même lot de lait on fabriquait en même temps d'autres fromages, non additionnés de levain. Ce lait était acidifié par addition d'acide lactique dilué jusqu'au $p H=5,3$. La concentration des ions d'hydrogène de ces fabrications de fromage variait de 5,1 $5,3 \mathrm{pH} 24 \mathrm{~h}$ après la fabrication et de 4,85 à 5,38 après trois mois d'affinage. La caractéristique plus détaillée des souches bactériennes utilisées, le degré de dégradation des protéines au cours de l'affinage et le goût et l'odeur des fromages ont été rapportés dans nos publications précédentes: Habaj, Poznañski, Rapczyñski, Rymaszewski (1966) et Poznañski, Habaj, Rymaszewski, Rapczyñski (1966).

Pour l'autre expérimentation, on a fait usage des mêmes souches, Str. Lactis 134 et 136, Str. diacetilactis 216 et 222, la culture mixte de ces quatre souches, et un levain généralement utilisé pour la fabrication de fromage, composé de streptocoques lactiques. Pour abaisser la teneur du fromage en lactose, et en même temps sa teneur en sels à pouvoir tampon (Birkkjaer, 1962), on ajoutait au grain de l'eau à la température de $32^{\circ} \mathrm{C}$ pendant le séchage, en proportion de 25 p. 100 du lait de fabrication. Les valeurs du $p \mathrm{H}$ variaient de $5,4-5,5 p \mathrm{H} 24 \mathrm{~h}$ après la fabrication, jusqu'à 5,8 - 6,0 $p \mathrm{H}$ après trois mois d'affinage. De même que pour la première expérimentation, des données analogues concernant la fabrication, l'affinage, le degré de dégradation des protéines, des caractéristiques de goût et d'odeur seront rapportées 
TABLEAC 1. -- Caractéristiques des propriétés qualitatives et de la dégradation des protéines de fromages avec la teneur en lactose basse et élevée.

\begin{tabular}{|c|c|c|c|c|c|c|c|}
\hline \multirow{2}{*}{$\begin{array}{l}\text { Sorte de fromage et } \\
\text { teneur en lactose }\end{array}$} & \multicolumn{3}{|c|}{$\mathrm{pH}$ du fromage } & \multicolumn{3}{|c|}{$\begin{array}{l}\text { Degré de dégradation des protéines } \\
\text { en pourcentage de } \mathrm{N} \text { total }\end{array}$} & \multirow{2}{*}{$\begin{array}{l}\text { Examen } \\
\text { organoleptique }\end{array}$} \\
\hline & & $\begin{array}{l}24 \text { heures } \\
\text { après la } \\
\text { fabrication }\end{array}$ & $\begin{array}{l}\text { après } 3 \text { mois } \\
\text { d'affinage }\end{array}$ & $\begin{array}{l}\text { N-soluble } \\
\text { à pH } 4,6\end{array}$ & $\begin{array}{l}\text { N-non- } \\
\text { protéique }\end{array}$ & $\begin{array}{l}\mathrm{N} \text { des } \\
\text { acides } \\
\text { aminés }\end{array}$ & \\
\hline \multirow{3}{*}{$\begin{array}{l}\text { Pâte demi-dure, teneur } \\
\text { en lactose élevée }\end{array}$} & 1 & 5,35 & 4,80 & 17,8 & 7,3 & 0,15 & \multirow{3}{*}{$\begin{array}{l}\text { lassants, farineux aci- } \\
\text { des/amers }\end{array}$} \\
\hline & 2 & 5,10 & 4,75 & 16,4 & 7,3 & 0,15 & \\
\hline & 3 & 5,10 & 4,75 & 16,7 & 7,5 & 0,15 & \\
\hline \multirow{2}{*}{$\begin{array}{l}\text { Pâte demi-dure, teneur } \\
\text { en lactose basse }\end{array}$} & 1 & 5,10 & 5,7 & 25,0 & 18,2 & 4,8 & \multirow{2}{*}{ correcte } \\
\hline & 2 & 5,10 & 5,6 & 23,0 & 18,3 & 2,9 & \\
\hline \multirow{3}{*}{$\begin{array}{l}\text { Fromages bleus, te- } \\
\text { neur en lactose éle- } \\
\text { vée }\end{array}$} & 1 & 5,51 & 7,7 & 34,4 & 23,6 & 4,4 & $\begin{array}{l}\text { légèrement amer, de } \\
\text { morge }\end{array}$ \\
\hline & 2 & 5,20 & 7,1 & 35,0 & 24,3 & 4,7 & $\begin{array}{l}\text { légèrement ammonia- } \\
\text { cal }\end{array}$ \\
\hline & 3 & 5,20 & 7,1 & 36,9 & 29,0 & 3,7 & acide, savonneux \\
\hline
\end{tabular}


dans notre publication à paraître: Poznañski, Rymaszewski, Habaj, Jakubowski.

Certaines différences étant observées dans des électrophorégrammes entre des fromages de la première fabrication et ceux de la seconde, nous avons décidé de fabriquer, dans des conditions du laboratoire et à l'aide d'un même levain de fromagerie, généralement utilisé, des fromages à pâte demi-dure qui eussent des propriétés semblables aux précédents, savoir, la teneur en lactose basse ou élevée. On a analysé aussi des "fromages bleus ", fabriqués, eux aussi, dans des conditions de laboratoire. La caractéristique de ces fromages est donnée dans le tableau 1.

\section{Méthodes}

Des fractions de protéines des fromages analysés étaient obtenues par précipitation à l'aide des ions de calcium suivant la méthode de Garnier (1964) que nous avions modifiée et adaptée pour le fromage (Poznañski, Rymaszewski, 1965). La fraction des protéines qu'on avait obtenue ainsi était ensuite diluée dans la solution de $\mathrm{NaOH}$ à $p \mathrm{H}$ 7-7,5 et lyophilisée. Après la lyophilisation, un poids défini des protéines était dilué dans la solution $7 \mathbf{M}$ de l'urée (concentration de protéine $-0,8$ p. 100).

On appliquait la technique d'électrophorèse d'après Wake et Baldwin (1961), modifiée par Schmidt (1964), au moyen de la chambre électrophorétique horizontale. Des fractions des caséines $\alpha_{s}$ et $\beta$ étaient obtenues par le procédé de la chromatographie sur colonne avec la DEAE-cellulose d'après Mercier, Maubois, Poznañski, Ribadeau-Dumas (1968).

Les électrophorégrammes ci-joints ne sont que des photocopies où les couleurs des fractions n'ont pas toujours été reproduites avec une intensité satisfaisante. Pour la discussion des résultats, la lecture était faite des phérogrammes originaux sur gel d'amidon.

\section{RESULTATS ET DISCUSSION}

Les électrophorégrammes de la fraction des protéines analysée dans le premier essai (Fig. 1) présentent les mêmes fractions peptidiques avec un rf identique, indépendamment de la culture qu'on a utilisée pour fabriquer les dits fromages. La fraction des protéines provenant du fromage qui a été fabriqué sans l'usage du levain, ne s'en différencie point en nombre et en répartition des fractions peptidiques. Une seule différence visible qu'on peut observer dans ces fromages concerne une fraction localisée dans la zone de la $\beta$ caséine, qui a disparu presque complètement. Nous avons supposé que l'activité des enzymes des bactéries lactiques était entravée par le $p \mathrm{H}$ du fromage, qui était trop bas pendant 


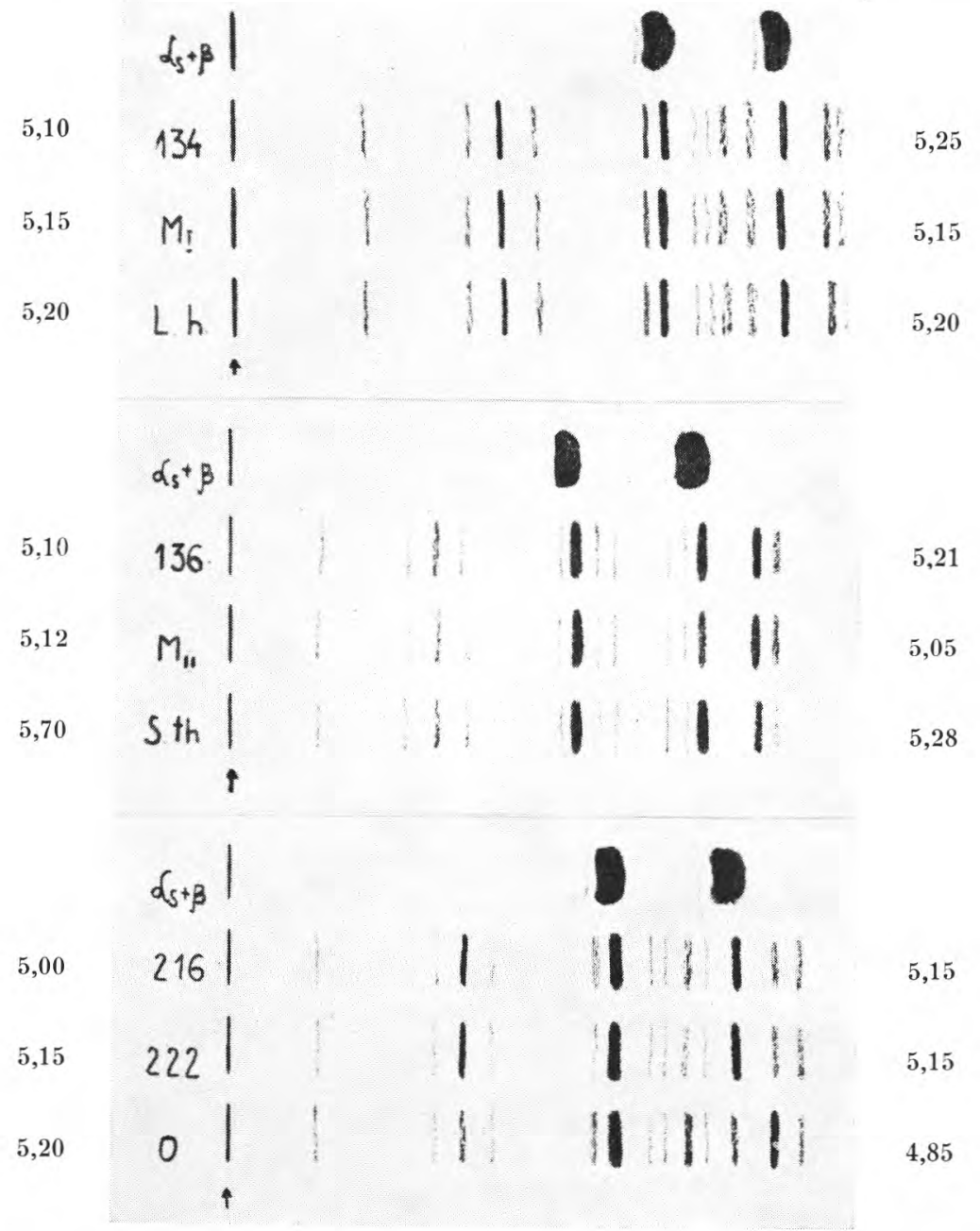

Fig. 1. - Répartition électrophorétique des protéines de fromage (précipitées avec $\mathrm{CaCl2}$ ) sur gel d'amidon.

Fromage de la sorte d'Edam du premier essai (la teneur en lactose élevée - changements insignifiants $\mathrm{du} p \mathrm{H}$ au cours de l'affinage). Les fromages étaient fabriqués avec du levain de cultures pures : Str.lactis 134, Str.lactis 136, Str.diacetilactis 216, Str.diacetilactis 222, la culture mixte de ces quatre souches $\mathbf{M}_{1}$, Str.thermophilus, L.helveticus, leur culture mixte $\mathrm{M}_{2}$, et non additionnés de levain - 0 .

$\alpha_{s}+\beta$ - fractions de caséine obtenues par le procédé de la chromatographie préparée sur colonne (Mercier, Maubois, Poznanski, RibadeauDumas, 1968). 
toute la période d'affinage. C'est pourquoi nous avons décidé d'analyser des fromages de la seconde expérimentation.

Pendant l'affinage de ce lot de fromages, des conditions étaient favorables pour l'activité enzymatique des bactéries lactiques: Poznañski, Rymaszewski, Habaj, Jakubowski (1968). Bien que certaines différences fussent observées entre des fromages du premier et de la seconde expérimentation dans une fraction peptidique moins distincte, et dans l'intensité de couleur de quatre fractions peptidiques ayant la mobilité électrophorétique assez faible, on n'a pourtant remarqué aucune différence dans la répartition des fractions peptidiques entre des fromages du second essai. Ces fractions se présentent d'une manière identique pour des fromages affinés avec des cultures pures, leur culture mixte et le levain de fromagerie (Fig. 2).

après 24 heures

$$
d s * 8 \mid
$$

5,42

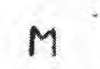<smiles>C1C[AsH][AsH]1</smiles>

5,45

5,40

5,50 $p \mathrm{H} \mathrm{du}$ fromage

après 3 mois

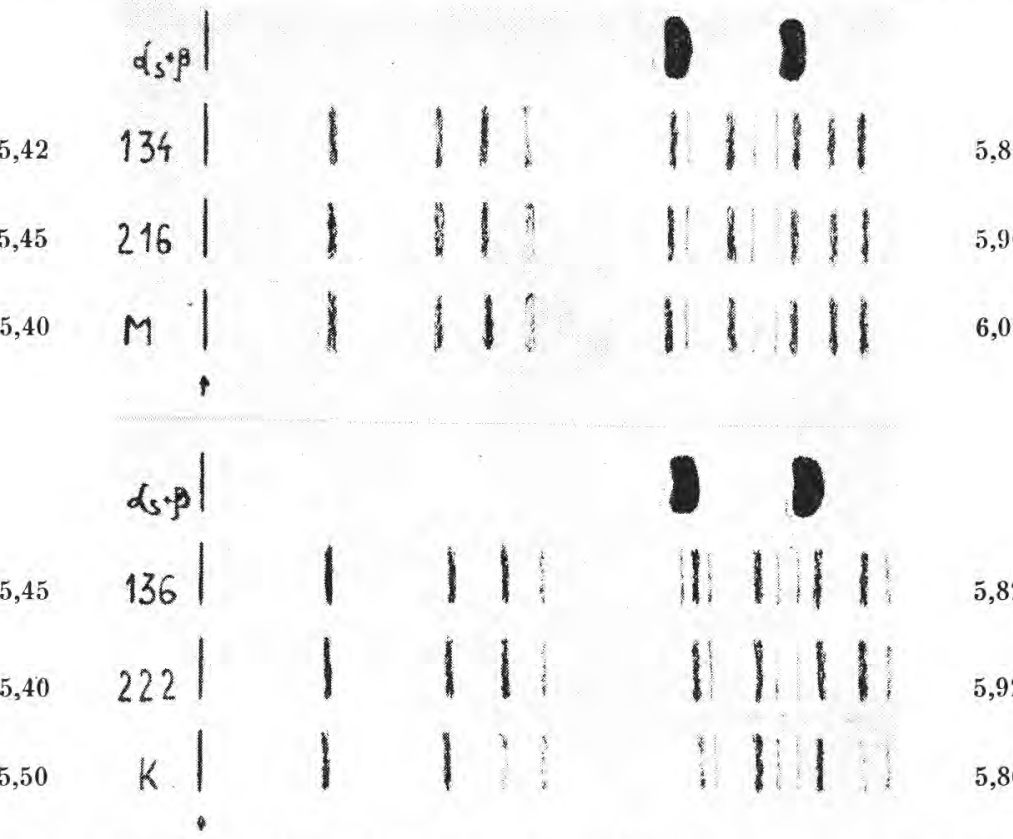

5,85

Fig. 2. - Répartition électrophorétique des protéines au fromage (précipitées avec $\mathrm{CaCl2}$ ) sur gel d'amidon.

Des fromages de la sorte d'Edam du second essai (teneur en lactose basse, abaissement considérable du $p \mathrm{H}$ pendant l'affinage). Les fromages étaient produits avec le levain des cultures pures : Str.lactis 134, Str.lactis 136, Str.diacetilactis 216, Str.diacetilactis 222, la culture mixte de ces quatre souches, ainsi que le levain ordinaire de fromagerie.

$\alpha_{c}+\beta-$ fractions de caséine obtenues par la méthode de préparation de la chromatographie sur colonne (Mercier, Maubois, Poznanski, RibadeauDumas, 1968). 
La répartition électrophorétique de lait dite fraction de protéines des fromages provenant du premier et du second lot semble confirmer la thèse que nous avons proposée dans nos expérimentations précédentes: la dégradation directe de la $\alpha_{5}$ et $\beta$-paracaséine est effectuée par la chymosine, tandis que les enzymes bactériens n'attaquent que des produits de cette dégradation accumulée par la présure - Poznañski, Lenoir, Mocquot (1965) ; Poznañski, Rymaszewski (1965); Habaj, Poznañski, Rapczyñski, Rymaszewski, (1966); Poznañski, Habaj, Rymaszewski, Rapczyñski (1966). Il semble plutôt impossible que les enzymes puissent dégrader la paracaséine d'une manière identique dans des conditions d'affinage tout à fait différentes, les souches mêmes présentant des différences manifestes dans leurs propriétés et leurs aptitudes protéolytiques.

On pourrait encore admettre que la composition des fractions de protéines qui ont été précipitées par des ions de calcium, contenait des fractions peptidiques insensibles à l'action des enzymes de la présure, ainsi qu'à celle des enzymes bactériens. Pourtant, prenant en considération le fait que la quantité des protéines qui avaient été précipitées par le $\mathrm{CaCl}_{2}$ variait dans des limites considérables (de 61,6 p. 100 pour le Str. diacetilactis 222 jusqu'à 75,6 p. 100 pour le Str. Lactis 134 et 46 p. 100 pour des fromages ne contenant que la présure), l'insensibilité des fractions peptidiques présentées à l'action des enzymes ne paraît guère probable. Des différences que nous avons constatées dans la mobilité électrophorétique des peptides obtenus par la digestion de la x-caséine avec la suspension des germes provenant de plusieurs souches de bactéries lactiques, aux valeurs du $p \mathrm{H}$ diverses, et puis par la digestion avec la trypsine à $p H \mathrm{H}$, ne paraissent point confirmer cette supposition - Rymaszewski, Poznañski (1968).

Ce ne sont que les différences, dans l'intensité de couleur de quatre fractions peptidiques à faible mobilité électrophorétique entre des fromages du premier essai et ceux du second, qui pourraient être attribuées à la différence du $p \mathrm{H}$ auquel la paracaséine était dégradée par les enzymes de la présure. On sait que l'activité de ces enzymes dépend du $\mathrm{pH}$.

En analysant des fromages à pâte demi-dure qui affinaient à $p \mathrm{H}$ bas (fromages $1,2,3$ ) le schéma électrophorétique ne diffère que bien peu de celui des fromages de la sorte Edam. Une seule différence s'y est montrée dans la mobilité de la quatrième fraction du côté de départ, ainsi que dans l'intensité de couleur de ces quatre fractions à faible mobilité électrophorétique. Il est intéressant d'observer que cette intensité est d'autant plus faible que le $\mathrm{pH}$ du fromage pendant l'affinage est plus bas. On trouve des différences plus remarquables dans le fromage $2 a$, qui était de la même sorte, mais qui affinait à morge (fig. 3 ) et qui a montré ine augmentation manifeste du $p H$ (de 5,1 à 5,6) pendant l'affinage. Comme on le voit bien sur l'électrophorégramme, le nombre des fractions peptidiques à la mobilité plus grande a diminué sensiblement, et on aperçoit une nouvelle fraction peptidique, ayant la propriété évidemment basique. 
La répartition des fractions peptidiques pour des fromages bleus $R_{1}, R_{2}$ et $R_{3}$ (fig. 3 ) diffère d'une manière essentielle de celle

après 24 heures

$$
p \mathrm{H} \text { du fromage }
$$

après 2 mois

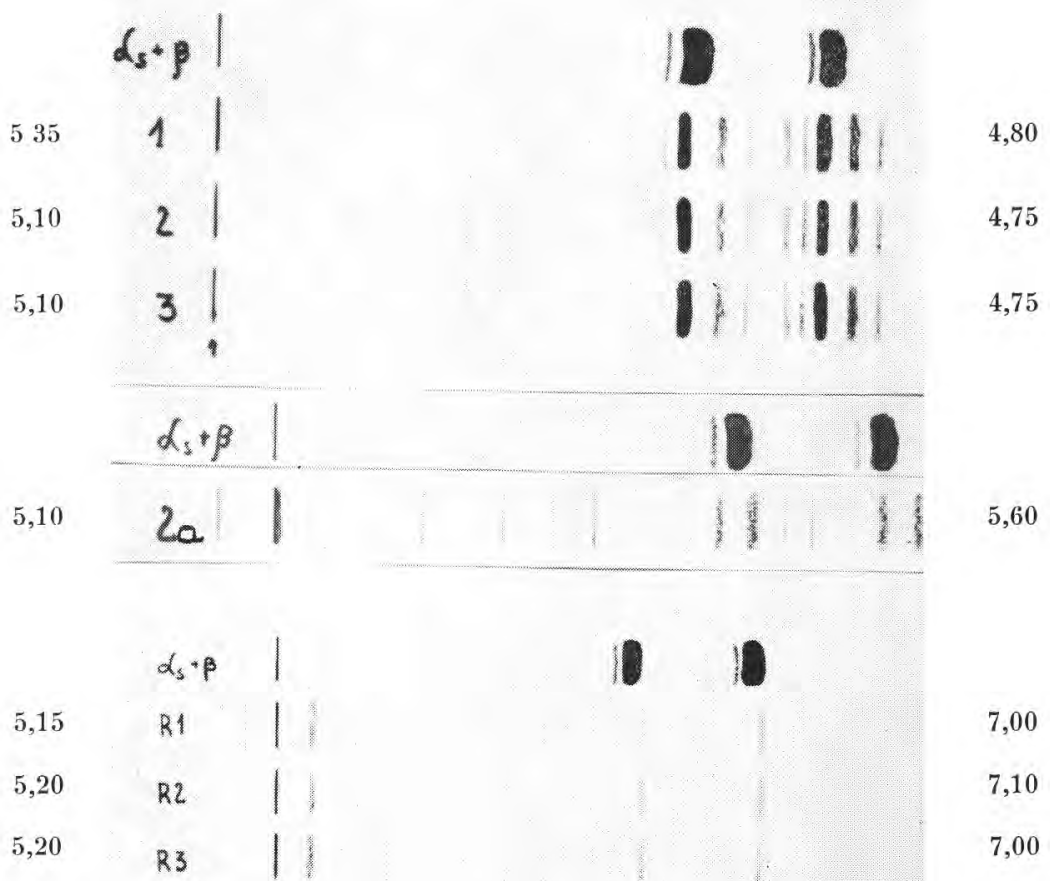

Fig. 3. - Répartition électrophorétique des protéines du fromage (précipitées avec $\mathrm{CaCl}$ ) sur gel d'amidon.

1, 2, 3 - Fromage à pâte demi-dure (teneur en lactose élevée, variations insignifiantes $\mathrm{du} \mathrm{pH}$ pendant l'affinage).

2 a - Fromage à pâte demi-dure (teneur en lactose basse, abaissement considérable du $p H$ pendant l'affinage). Fromage affinant sous la morge. $\alpha+\beta-$ fractions de caséine obtenues par la méthode de la chromatographie préparée sur colonne (Mercier, Maubois, Poznanski, RibadeauDumas, 1968).

des autres fromages examinés. Des fractions natives de la caséine $\alpha_{s}$ et $\beta$ ont subi une dégradation presque complète dans des fromages bleus; il y manque aussi des fractions peptidiques à grande mobilité électrophorétique, si nombreux dans les fromages précédemment décrits. Ce ne sont que quatre fractions à faible mobilité électrophorétique, et une fraction distinctement basique qu'on retrouve dans le fromage $R_{1}$, qui se rencontrent dans les deux 
groupes de fromages. Au contraire, des fromages précédemment discutés, le schéma électrophorétique des protéines de fromages bleus semble indiquer que la dégradation de la paracaséine $\alpha_{5}$ et $\beta$ a été effectuée non seulement par la présure, mais aussi par des enzymes du Penicillium roqueforti. De même, le schéma électrophorétique du fromage qui affinait à l'aide de la morge laisse supposer la dégradation de la $\alpha_{5}$ et $\beta$-paracaséine par des bactéries contenues dans la morge (fig. 3,2 a).

L'interprétation des résultats que nous avons obtenus est rendue un peu difficile par le fait que l'intensité de couleur d'une fraction peptidique ne dépend pas toujours de la quantité du peptide lui-même. Comme on le sait, l'aptitude du noir amido de lier des protéines et des peptides dépend de la quantité des résidus d'acides aminés: la lysine, l'arginine et l'histidine - Manson (1966), et des enzymes protéolytiques modifient considérablement l'aptitude du noir amido de lier des protéines attaquées - Poznañski, Habaj, Rymaszewski, Rapczyñski (1966); Rymaszewski, Poznañski (1968). La mise au point de l'électrophorèse préparatoire à cet effet contribuerait sans doute à expliquer plus clairement ce phénomène complexe. Il ressort encore de nos expérimentations

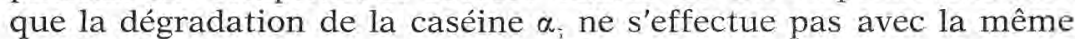
facilité à toutes les valeurs de $p \mathrm{H}$, ce qui a été remarqué par Payens (1958), Poznañski (1964). Elle dépend plutôt des conditions d'affinage et de la sorte de fromage - Lindqvist et Stortgards (1955).

Pour finir, nous n'avons remarqué aucun rapport entre une fraction ou des fractions des peptides répartis et des propriétés de goût des fromages. Ce problème mérite une investigation à part.

(Reçu pour publication, le 17 septembre 1968).

\section{$R$ és um é}

On s'est servi de l'électrophorèse sur gel d'amidon pour étudier le degré de dégradation des protéines du fromage qui ont été précipitées avec des ions de calcium à la concentration de $0,03 \mathrm{M}$. On a analysé des fromages affinés de la sorte d'Edam qui avaient été fabriqués avec des cultures pures suivantes ou leurs cultures mixtes: Str. lactis 134 et 136, Str. diacetilactis 216 et 222, la culture mixte de ces quatre souches $\left(\mathrm{M}_{1}\right)$, Str. thermophilus, L. helveticus et leur culture mixte $\left(\mathrm{M}_{2}\right)$. Des fromages étaient fabriqués avec une teneur en lactose basse ou élevée de la pâte fraîche du fromage, ce qui décidait des limites du $p \mathrm{H}$ durant toute la période d'affinage. Des fromages expérimentaux à pâte demi-dure, avec la teneur en lactose, eux aussi, basse ou élevée, ont été analysés complémentairement. 
Des électrophorégrammes de la fraction des protéines du fromage analysée ont montré des fractions peptidiques dans tous les lots des fromages de la sorte d'Edam, indépendamment des souches de bactéries lactiques utilisées pour la fabrication, des différences du $p \mathrm{H}$ pendant l'affinage et de celles de l'odeur et de la saveur. On a pourtant constaté des différences évidentes dans des fractions peptidiques des protéines qui avaient été précipitées des fromages bleus. Des résultats que nous avons obtenus semblent confirmer la thèse que nous avons proposée dans nos études précédentes: la dégradation directe de la paracaséine $\alpha_{5}$ et $\beta$ était effectuée par la chymosine, des enzymes attaquant les produits de dégradation de la caséine que la présure avait accumulés.

\section{S u m m a r y}

Characteristics of cheese proteins as precepitated by calcium ions when using starch gel electrophoresis.

Starch gel electrophoresis was used to investigate the breakdown extent of cheese proteins, as precepitated by calcium ions at concentration of $0,03 \mathrm{M}$. Analysis was made of ripened cheeses of Edam type which had been manufactured with following singlestrain cultures or their mixed-strain cultures: Str. lactis 134 and 136, Str. diacetilactis 216 and 222, the mixed-strain culture of this four strains $\left(\mathrm{M}_{1}\right)$, Str. thermophilus and Str. helveticus, and their mixed-strain culture $\left(\mathrm{M}_{2}\right)$. The cheeses were manufactured with both low and high lactose contents in fresh cheese body what determined the $p \mathrm{H}$ range throughout the ripening. In addition, some cheeses of semi-hard type and blue cheeses were manufactured, with both high and low lactose content.

From the electrophoregrams of the cheese protein fraction analysed it appears that peptide fractions are identical in all lots of cheese of Edam type, irrespective of lactic strains, as used for their manufacture, differences of $p \mathrm{H}$ at which the cheese was ripening, as well flavour differences. Visible differences in peptide fractions were found for proteins which had been precepitated from blue cheeses. The results obtained seem to support the suggestion which was made in our previous studies: that the immediate breakdown of $\alpha_{5}$ and $\beta$ casein was performed by chymosine, enzymes attaking breakdown products which had been accumulated by action of rennin.

\section{BIBLIOGRAPHIE}

Assenat (L.). 1967. Contribution à l'étude d'urée méthode d'identification des laits et fromages au moyen de l'électrophorèse sur gel de polyacrylamide. Le Lait, 467 et 468,393 et 475 . 
BIRKKJAER (H.E.) 1962. Influence of the lactic acid fermentation upon the qualitities of the cheese. XVI Int. Dairy Congress, vol. B., 79.

Christ voN (W.) 1965. Caseinveranderungen Während der Käsereifung - Electrophoretische Untersuchungen in Polyacrylamidgelen. Milchwiss, 20, 133.

EMmons (D.B.) et all., 1962. Effect of strain of starter culture and of manufacturing procedure on bitterness and protein breakdown in Cheddar cheese. J. Dairy Sci. 45, 322.

Garnier (J.). 1964. Etude cinétique de la protéolyse par la présure de la caséine Kappa. Inst. National de la Rech. Agronomique, Série A, No 3932, No d'ordre 4753.

Habaj (B.), PozNANSKi (S.), RAPCZynsKi (T.), RymaszewsKi (J.). 1966. Effect of different strains of starter bacteria on the quality and texture of edam cheese. XVII Int. Dairy Congress, Sec. D 2, 471.

Lindouist (B.), Storgards (T.). 1959. Changes of casein during cheese ripening. XV Int. Dairy Congress, 2, 3, 679.

Manson (W.). 1966. The reaction of casein with the basic dye - safranine. XVIII Int. Dairy Congress, Sec. D 2, 193

Mercier (J. C.), Maubois (J. L.), Poznanski (S.), Ribadeau-Dumas, 1968. Fractionnement préparatif des caséines de vache et de brebis par chromatographie sur DEAE cellulose, en milieu urée et 2-mercaptoéthanol. Bull. Soc. Chim. Biol. 3, 50, 521.

Ney (K.H.), Wirotama (J.P.G.) und SeItz (J.). Die Untersuchung Käsemittels Polyacrylamidgel - Elektrophorese. XVII Int. Dairy Congress, 1966. Sec. D 1, 283.

P.IYENS (T. A. J.). Elektroforetisch onderzoek van de Eiwitafbraak Tijdens de Kaasrijping. Nederlands Melk-en-Zuireltijdschrift, 1958, 2, 99.

PozNANSKi (S.). Abbau der Wasser und Alkohol unloslichen Stickstoff - Verbindungen bei der Reifung von Edamer Käsen. Milchwiss, 1964, 19, 425.

Poznanski (S.), Lenoir (J.), Mocovot (G.). La protéolyse de la caséine par les enzymes intracellulaires de certaines bactéries. Le Lait, 1965, 441-442, 3.

Poznanski (S.), Rymaszewski (J.) Proteolysis during the ripening of Edam cheese with the participation of some bacteria strains. Part I and II, Milchwiss, 1965, 20, 14 and 20, 295.

PoZnANSKI (S.), HABAJ (B.), RYMASZEWSKI (J.), RAPCZYNSKI (T.). Influence of different starter cultures on the protein breakdown in Edam cheese, XVII Int. Dairy Congress, 1966. Sec. D 2, 555.

Poznanski (S.), Rymaszewski (J.), Habaj (B.), Jakubowski (J.). Degradacja bialek w serze przy udziale pewnych szczepów bakterii mlekowych opornych na bakteriofagi. Zeszyty Naukowe WSR Olsztyn 1968. (sous-presse).

RYMASZEWSKI (J.), POZNANSKI (S.). The breakdown of Kappa-casein components of lactic acid bacteria. Bull. de l'Académie Polonaise des Sciences. Série des Sciences Biologiques, 1698. (sous-presse).

Schmidt (D. G.) Starch-gel elektrophoresis of K-casein. Biochim. Biophys. Acta, 1964, 90 411.

WAKE (R.), BALDWIN (R.L). Analysis of casein fractions by zone elektrophoresis in concentrated urea. Biochim. Acta, 1961, 47, 225. 